
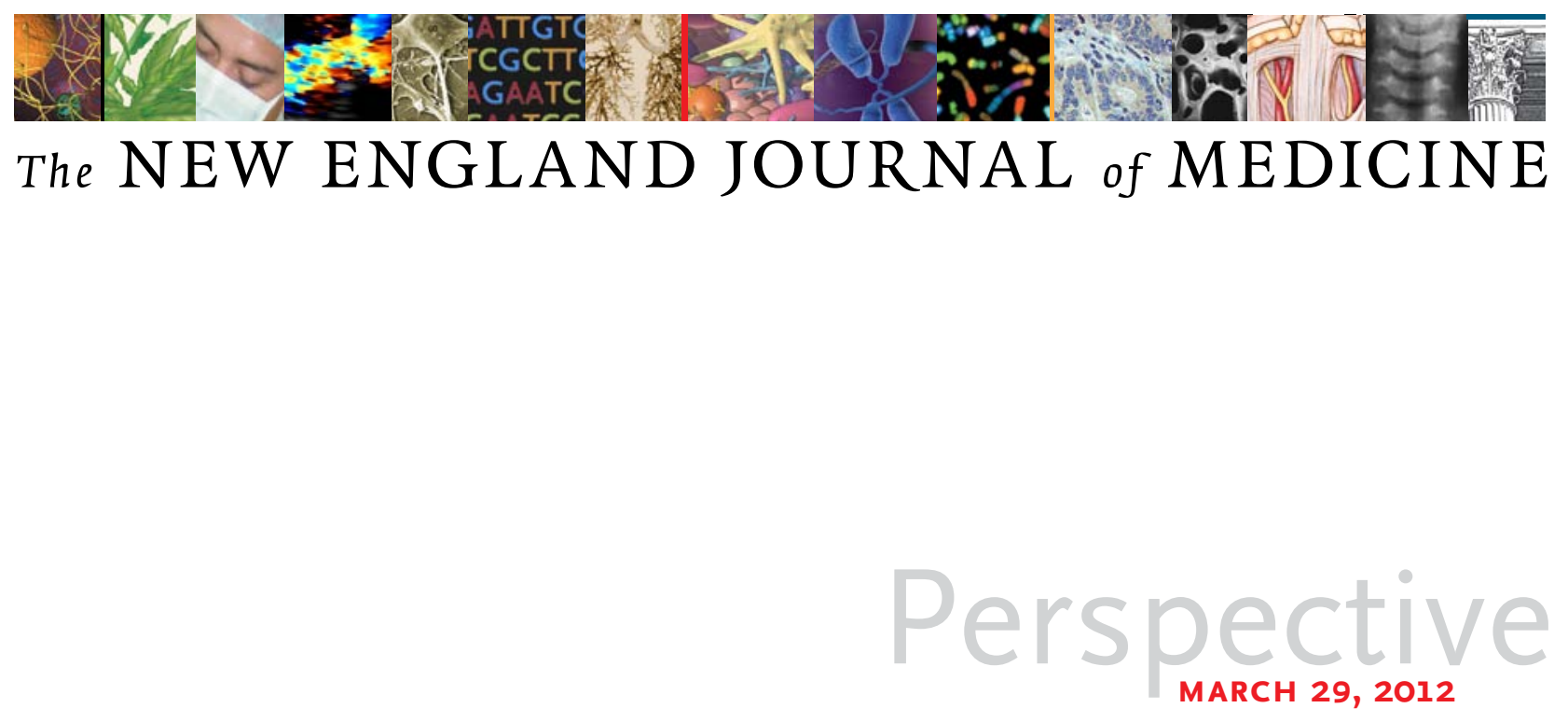

\title{
Tropical Lymphedemas - Control and Prevention
}

David H. Molyneux, Ph.D., D.Sc.

Related article, p. 1200

here are two principal causes of elephantiasis,
or lymphedema, in the tropics. The most common cause and a significant public health problem is lymphatic filariasis due to the parasitic nematode

Wuchereria bancrofti (and, in Asia, Brugia malayi and B. timori), which is transmitted by mosquitoes. The second principal cause is podoconiosis.

Lymphatic filariasis is endemic in some 72 countries throughout the tropics (see map), and an estimated 1.39 billion people live in areas of risk. ${ }^{1}$ An estimated 40 million people have stigmatizing and disabling clinical manifestations of the infection: 15 million have elephantiasis, and some 25 million men have genital swelling, usually scrotal hydrocele. ${ }^{1}$ The current strategy for stopping transmission is the annual mass administration of donated drugs - albendazole and ivermectin in areas of Africa where onchocer- ciasis is coendemic, and diethylcarbamazine and albendazole elsewhere.

The Global Program to Eliminate Lymphatic Filariasis (GPELF), begun in 2000, is aimed at eliminating transmission of the infection and reducing associated illnesses. Through annual mass distribution of drugs, substantial progress has been made in 12 years toward the elimination target set by the GPELF, with about 466 million people treated in 2010. The economic benefits accrued through the program from 2000 through 2008, including the ancillary benefits of deworming and the impact on scabies, have been calculated at $\$ 24$ billion. Costs per year for control of lymphatic filariasis range from $\$ 0.06$ to $\$ 1.00$ per person, depending on the setting; as with other neglected tropical diseases, costs of preventive chemotherapy are generally less than $\$ 0.50$ per person per year. Therefore, prevention of lymphatic filariasis ranks among the most cost-effective global health interventions, along with deworming and onchocerciasis prevention, as measured in cost per disability-adjusted life-year averted. ${ }^{2}$

Lymphatic filariasis is one of 17 neglected tropical diseases that afflict the world's poorest people, but another even more neglected condition causing elephantiasis has been highlighted in recent years - podoconiosis, which affects some 4 million people in Africa, Latin America, and a few areas of Asia $^{3}$ (see map). For many years, podoconiosis was relegated to the small print of even specialist tropical medicine 


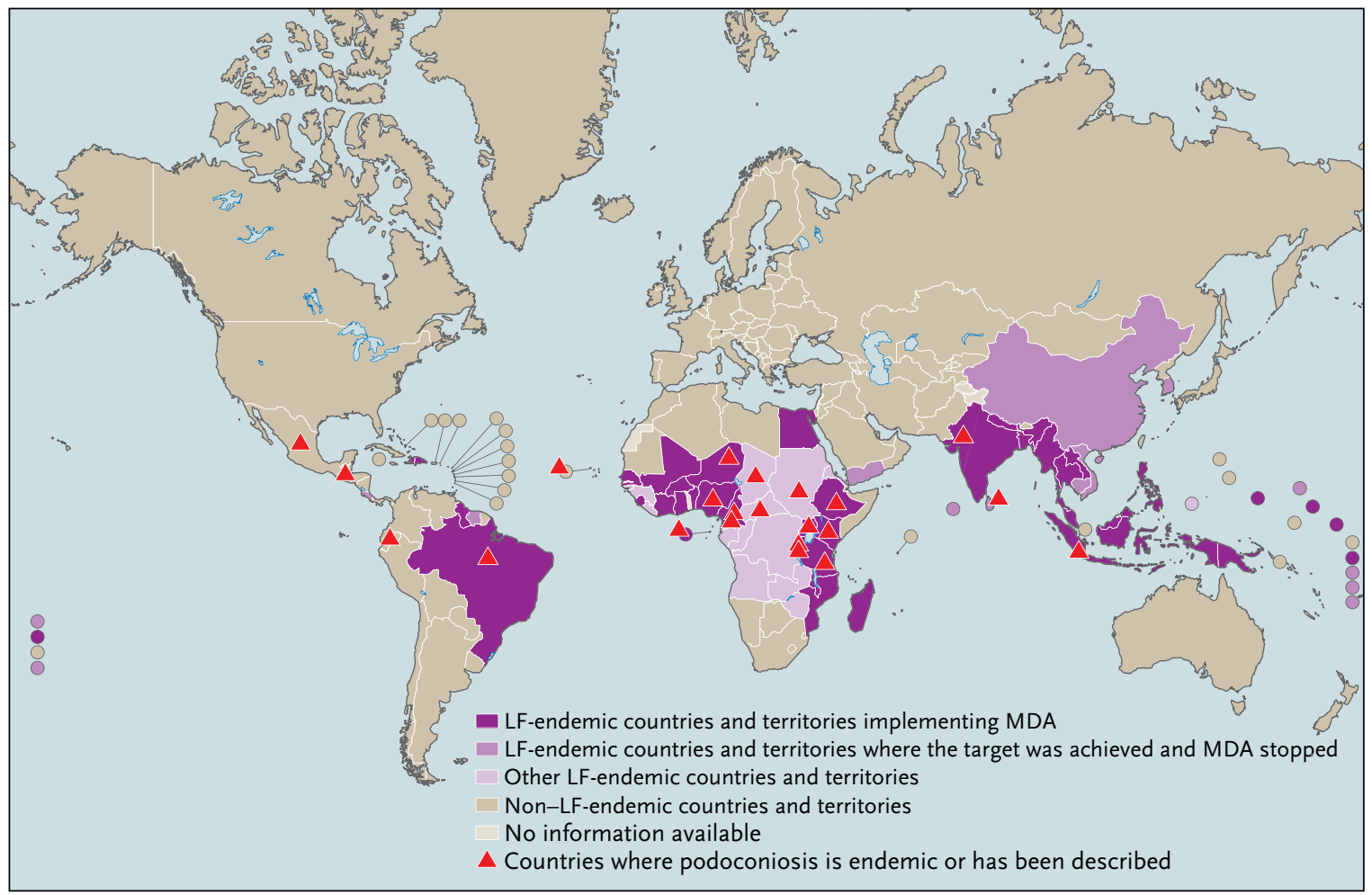

Global Distribution of Lymphatic Filariasis (LF) and Podoconiosis and Status of Mass Administration of Drugs (MDA), 2009.

Data are from the World Health Organization. Dots represent various island nations.

textbooks, but it has emerged as an important cause of illness in communities where people are exposed to irritant, red-clay, volcanic soil. Microsilica particles from the soil enter the body through the skin of the feet and cause chronic obstruction of the lymphatics.

The article by Tekola Ayele et al. in this issue of the Journal (pages 1200-1208) highlights the role of genetics in the predisposition to this condition. The authors found a significant association of podoconiosis with particular genetic variants in the HLA class II region, and they found that certain single-nucleotide polymorphisms increase the risk of clinical disease by a factor of two to three. Affected people have symptoms similar to those associated with filarial lymphedema: adenolymphangitis, plantar edema, moss- like papillomas ("mossy foot"), and multiple skin nodules, with progression to a hard, fibrotic leg (see photo). Podoconiosis must be distinguished from filarial lymphedema - it is found at altitudes higher than those at which mosquitoes can transmit filarial infection (a maximum of about $1500 \mathrm{~m}$ ). The study by Tekola Ayele et al. highlights the importance of examining the human genetics of disease in resource-poor areas to enable the targeting of interventions, which in this case are as simple as providing shoes to people with a family history of podoconiosis. Previous work in Ethiopia revealed the social and economic effects of the disease affected people have a markedly reduced earning capacity.

More than 705 million people received integrated preventive che- motherapy for at least one neglected tropical disease in 2009, and 702 million to 715 million did so in $2010 .{ }^{4}$ More than 800 million people are treated annually for some form of helminth disease or trachoma, according to the World Health Organization, and more than half of them are treated for lymphatic filariasis. Expansion of the lymphatic filariasis program in Central Africa, and in the Democratic Republic of the Congo and Cameroon in particular, is constrained by the risk of severe adverse reactions, manifested as encephalopathy, to treatment with ivermectin among persons with high microfilarial loads of the tropical eye worm Loa loa. This problem was initially identified in onchocerciasis-control programs during the mid1990s and has prevented large- 
scale treatment for lymphatic filariasis because of the potential coendemicity of $W$. bancrofti and L. loa in Central Africa.

Whereas annual mass drug administration relies on the ability of drugs to reduce microfilaria loads to levels at which transmission is reduced, a high-priority need is for a drug that kills or permanently sterilizes the adult worms - a macrofilaricide. It is known that some filarial nematodes contain the endosymbiotic bacteria wolbachia, which have become targets for chemotherapy, since they have also been implicated as contributors to the pathogenesis of inflammatory disease. Doxycycline is effective when given daily over a 6-week period, since it kills or sterilizes adult filaria of both Onchocerca volvulus and $W$. bancrofti. Ongoing research is seeking to reduce the duration of antibiotic treatment and to find a less toxic product that might be given over a shorter period and be amenable to administration by the communities themselves, as treatments for onchocerciasis and lymphatic filariasis are. Although the current drugs (albendazole, ivermectin, and diethylcarbamazine) are designed to be given for the duration of the adult worms' life (an estimated 5 to 8 years) and at a coverage level of $75 \%$ of the population in all geographic areas where they are endemic, they also have an effect on clinical disease: they reduce the frequency of filarial fevers, the size of scrotal hydrocele, and in children, subclinical lymphatic damage.

One of the challenges for the GPELF has been that diseasecontrol programs that address the needs of patients with existing clinical lymphedema or hydro- cele have lagged behind mass drug distribution. It is critical that this gap be addressed. The drivers of success will be maintenance of high coverage, provision of resources for monitoring and evaluation, and efforts to understand the complexity of the interactions among coendemic conditions such as lymphatic filariasis, onchocerciasis, and soil-transmitted helminths; control or elimination interventions for all these conditions benefit from donated drugs. The success of lymphatic filariasis programs in Africa will also be influenced by the extent of coverage with bed nets impregnated with long-lasting insecti-

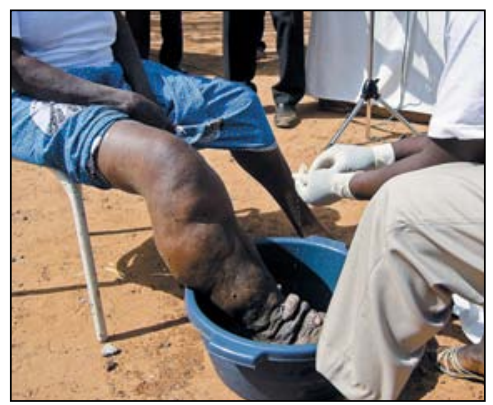

Legs of a Man with Lymphatic Filariasis.

cide, which, if distributed widely, could probably reduce the required number of annual mass drug administrations.

Over the past decade, there has been remarkable progress in addressing lymphatic filariasis as a global problem. Several billion doses of drugs have been donated and delivered, and they have had the additional benefit of effective deworming. Still, programs providing surgery for scrotal hydrocele need to be scaled up, and access to lymphedema-management programs must be increased. The lymphatic filariasis community is aware of the need for improved therapy, especially a macrofilaricide, as well as the challenges of scaling up programs in countries that are recovering from conflict and civil unrest and of the need to expand disease control, address the L. loa issue, enhance coverage, and monitor and evaluate impact. Certification of the absence of transmission awaits detailed transmission-assessment surveys. Research into improving the costeffectiveness of elimination programs will continue, and their lessons need to be not only learned but also applied.

The two tropical lymphedemas, filariasis and podoconiosis, are now on the radar screen for global health. The two conditions share clinical features and challenges that are similar to highly disabling conditions affecting the most disadvantaged communities, and they inflict similar social problems. It may be possible, however, to eliminate both diseases. The idea that impoverished people might wear shoes may seem self-evident, but being able to target the people at greatest risk represents considerable progress.

Disclosure forms provided by the author are available with the full text of this article at NEJM.org.

From the Centre for Neglected Tropical Diseases, Liverpool School of Tropical Medicine, Liverpool, United Kingdom.

1. Global programme to eliminate lymphatic filariasis: progress report on mass drug administration, 2010. Wkly Epidemiol Rec 2011;86:377-88.

2. Ottesen EA, Hooper PJ, Bradley M, Biswas $\mathrm{G}$. The global programme to eliminate lymphatic filariasis: health impact after 8 years. PLoS Negl Trop Dis 2008;2(10):e317.

3. Davey G, Newport M. Podoconiosis: the most neglected tropical disease? Lancet 2007;369:888-9.

4. Integrated preventive chemotherapy for neglected tropical diseases: estimation of the number of interventions required and delivered, 2009-2010. Wkly Epidemiol Rec 2012;87:17-27.

Copyright @ 2012 Massachusetts Medical Society. 